\title{
BRAQUITERAPIA INTERSTICIAL PARA RECIDIVAS DE CÂNCER DE COLO UTERINO PÓS-RADIOTERAPIA*
}

\author{
Antonio Carlos Zuliani de Oliveira ${ }^{1}$, Sérgio Carlos Barros Esteves ${ }^{2}$, Luiz Fernando Andrade Feijó ${ }^{2}$, \\ Eduardo Komai Tagawa ${ }^{2}$, Maércio de Oliveira Cunha ${ }^{2}$
}

Resumo OBJETIVO: Analisar a resposta e toxicidade da braquiterapia de alta taxa de dose (BATD) intersticial para carcinoma do colo do útero com recidiva pélvica pós-radioterapia. MATERIAIS E MÉTODOS: Entre 1998 e 2001, 11 pacientes com carcinoma de colo de útero e que tiveram recidiva pélvica pós-radioterapia receberam BATD intersticial. Idade: 41 a 71 anos (média: 56,5 anos); estádios (FIGO): IIA, IIB, IIIB e IVA. Nove $(82 \%)$ pacientes tinham carcinoma de células escamosas e duas $(18 \%)$, adenocarcinoma. Dose total de BATD: 20-30 Gy, em frações de 4-5 Gy. 0 seguimento variou de dois a 54 meses (média: 22,5 meses), através de exame físico periódico (três meses). Uma paciente faleceu sem avaliação de resposta. RESULTADOS: Dez pacientes (91\%) tiveram resposta clínica completa, com duração de três a 46 meses (média: 18,9 meses). Três pacientes estão livres de doença (27\%), duas estão vivas com doença (18\%), três morreram $(27 \%)$ e de três se perdeu o seguimento após nova recidiva $(27 \%)$. A toxicidade para o trato urinário foi de 9\% (uma paciente - grau III). CONCLUSÃO: A BATD intersticial é uma abordagem alternativa e viável para pacientes selecionadas que tiveram recidiva pós-radioterapia. Foi possível obter altas taxas de resposta com baixa toxicidade, considerando-se o grupo estudado, o tempo de seguimento e a re-irradiação.

Unitermos: Câncer de cérvix recidivado; Radioterapia; Braquiterapia de alta taxa de dose; Câncer ginecológico.

Abstract Interstitial high-dose rate brachytherapy for recurrent cervical cancer after radiation therapy.

OBJECTIVE: To evaluate the response and potential toxicity of fractionated interstitial high-dose rate (HDR) brachytherapy for post-radiation pelvic recurrence in cervical cancer. MATERIALS AND METHODS: From 1998 to 2001, 11 patients aged 41 to 71 years with cervical carcinoma stages II to IV who presented pelvic recurrence after radiation therapy were treated wit interstitial HDR. Nine of these patients (82\%) had squamous cell carcinoma and two had adenocarcinoma. Radiation consisted of $20 \mathrm{~Gy}$ to $30 \mathrm{~Gy}$ delivered in fractions of $4 \mathrm{~Gy}$ to $5 \mathrm{~Gy}$ over three days. The median follow-up was 22.5 months ( 2 to 54 months), with periodic physical examinations (three months interval). One patient died without evaluation of the response. RESULTS: Ten patients $(91 \%)$ presented complete clinical response, three patients $(27 \%)$ were disease free, two were alive with disease, three $(27 \%)$ died of cancer and three $(27 \%)$ were lost in the follow-up after the second recurrence. Urinary tract toxicity grade III was $9 \%$ (one patient). CONCLUSION: Interstitial HDR brachytherapy is an alternative approach to selected patients with recurrent cervical carcinoma after radiation therapy. High response rates were achieved with low toxicity taking into account the studied group, time of follow-up and re-irradiation.

Key words: Recurrent cervical carcinoma; Radiation therapy; High-dose rate brachytherapy; Gynecological cancer.

\section{INTRODUÇÃO}

Segundo dados do Instituto Nacional de Câncer (INCA), a neoplasia do colo uterino é a terceira mais freqüente entre as mulheres, ficando atrás do câncer de mama e de pele não-melanoma ${ }^{(1)}$.

* Trabalho realizado no Centro de Atenção Integral à Saúde da Mulher (CAISM) da Universidade Estadual de Campinas (Unicamp), Campinas, SP.

1. Médico Estagiário do CAISM-Unicamp.

2. Médicos Radioterapeutas do CAISM-Unicamp.

Endereço para correspondência: Dr. Sérgio Esteves. Departamento de Radioterapia, CAISM-Unicamp. Rua Alexander Fleming 101, Cidade Universitária Zeferino Vaz. Campinas, SP, 13083 970. E-mail: estevesrt@uol.com.br

Recebido para publicação em 22/11/2002. Aceito, após revisão, em 24/5/2004.
As pacientes que são encaminhadas para radioterapia apresentam doença em estádios avançados. Este fato determina altos índices de recidiva loco-regional.

A incidência de recidiva pélvica pósradioterapia exclusiva se distribui da seguinte maneira, de acordo com o estádio clínico: estádio IB, 5\% a $8 \%$; IIA, $15 \%$ a $20 \%^{(2-7)}$; IIB, $18 \%$ a $39 \%^{(8-11)}$; IIIB, $38 \%$ a $50 \%^{\left({ }^{(9-13)}\right.}$; IVA, $60 \%$ a $80 \%{ }^{(\mathbf{1 4})}$.

A abordagem terapêutica dessas recidivas é difícil, controversa e, em geral, de pouca eficácia.

A cirurgia de resgate pode ser oferecida a pacientes com doença muito limitada e central, caso apresentem condições clí- nicas ${ }^{(15)}$. A cirurgia radical pós-radioterapia é acompanhada de mortalidade em alguns casos, morbidade importante em muitos e perda substancial de estrutura e função em todas as pacientes operadas ${ }^{(16,17)}$. A aplicabilidade da cirurgia de resgate, geralmente exenterativa, é limitada pela aceitação de médico e paciente e por parâmetros clínicos.

A re-irradiação é mais bem tolerada agudamente, tem pouca ou nenhuma mortalidade operatória (no caso de braquiterapia) e freqüentemente preserva a estrutura e função de órgãos pélvicos. Entretanto, efeitos tardios graves são comuns, particularmente em séries antigas com uso de 
equipamentos e técnicas inferiores aos padrões atuais ${ }^{(\mathbf{1 8 , 1 9 )}}$.

A literatura mais recente reporta resultados melhores com a re-irradiação, particularmente com maior ênfase nas técnicas de braquiterapia.

Puthawala et al. usaram implantes intersticiais em 40 pacientes com recidiva pélvica de diferentes tumores pós-radioterapia: 26 receberam os implantes durante laparotomia exploradora, duas recusaram cirurgia e 12 não foram operadas. Vinte e sete $(67 \%)$ apresentaram resposta completa, 13 (33\%) tiveram sobrevida livre de doença com dois anos de seguimento mínimo, seis $(15 \%)$ apresentaram toxicidade grau IV (necrose e fístula) ${ }^{(\mathbf{2 0})}$.

Randall et al. trataram com implantes intersticiais 13 pacientes com neoplasias ginecológicas recorrentes pós-radioterapia, sendo seis carcinomas de endométrio recorrentes, quatro carcinomas de colo recorrentes e três novos primários de vagina. Trataram seis pacientes com implantes temporários de irídio-192, doses de 40-55 Gy, 0,35-0,50 Gy/hora, e sete pacientes com implantes permanentes de paládio103 e ouro-198, com doses de 30-90 Gy. Nove $(69 \%)$ pacientes apresentaram resposta completa, seis $(46 \%)$ continuaram sem evidência de doença, com seguimento de 24 a 71 meses (média de 59 meses), e uma $(7,7 \%)$ paciente apresentou toxicidade grau IV (fístula reto-vaginal) ${ }^{(21)}$.

O objetivo deste estudo foi analisar, retrospectivamente, a resposta e a toxicidade da braquiterapia de alta taxa de dose (BATD) com implantes intersticiais para recidiva local de neoplasia do colo de útero pós-radioterapia.

\section{MATERIAIS E MÉTODOS}

Durante o período de novembro de 1998 a setembro de 2001, 11 pacientes com recidiva pélvica isolada de carcinoma do colo do útero, todas com radioterapia prévia, foram submetidas à braquiterapia intersticial.

As recidivas ocorreram no período de quatro meses a 18 anos pós-radioterapia.

Ao tratamento, a idade média do grupo era de 56,5 anos (41 a 71 anos).

Por ocasião do diagnóstico inicial, os estádios eram IIA (18\%), IIB (9\%), IIIB
(45\%) e IVA (9\%). Duas pacientes tratadas em outros serviços há mais de dez anos não tiveram o estadiamento inicial identificado (Tabela 1).

Quanto ao tipo histológico, nove (82\%) pacientes apresentavam carcinoma espinocelular e duas (18\%), adenocarcinoma.

As pacientes apresentaram recidivas localizadas no colo do útero, vagina e paramétrios.

Três pacientes receberam associação de teleterapia de forma localizada, dose de 34-36 Gy, de um a três meses antes da braquiterapia intersticial.

Três pacientes, com recidiva pós-radioterapia, haviam sido previamente tratadas com cirurgia. Submetidas à histerectomia total abdominal, apresentaram nova recidiva local. Foram então submetidas à braquiterapia intersticial como segunda tentativa de resgate.
A BATD foi realizada através de agulhamento perineal guiado por ultra-som, sob anestesia raquidiana. Para esse procedimento foram utilizadas de cinco a 20 agulhas.

O intervalo entre as frações foi de seis horas em nove pacientes, o que levou à internação durante o tratamento. Duas pacientes receberam as aplicações em intervalos de sete dias, tendo sido tratadas em regime ambulatorial.

A dose total variou de 20 Gy a 30 Gy, em quatro a seis frações, de 4 Gy a 5 Gy, sempre prescritas na isodose de $85 \%$.

Os índices de uniformidade variaram de 1,7 a 2,11 (média de 1,8), e a relação entre os volumes das curvas de isodose de $150 \%$ e $100 \%$ variou de 13,9 a 28,6 (média de 22,2) (Tabela 2).

O seguimento variou de dois a 54 meses (média de 22,5 meses), com exames

Tabela 1 Descrição do grupo de pacientes, do primeiro tratamento e data da recidiva.

\begin{tabular}{|l|l|l|l|l|l|l|}
\hline Idade & $\begin{array}{c}\text { Estádio } \\
\text { inicial }\end{array}$ & \multicolumn{1}{|c|}{ Teleterapia } & Braquiterapia & $\begin{array}{c}\text { Término } \\
\text { do primeiro } \\
\text { tratamento }\end{array}$ & Recidiva & Histerectomia \\
\hline 52 & IIB & 45 Gy & BATD $4 \times 7$ Gy & Nov./1997 & Jan./2001 & Não \\
48 & IIIB & $45+14,4$ Gy & BATD $4 \times 7$ Gy & Mar./1998 & Jan./1999 & Não \\
43 & IIIB & $45+14,4$ Gy & BATD $4 \times 7$ Gy & Abr./2000 & Out./2000 & Não \\
51 & IVA & $46,8+12,6$ Gy & BATD $4 \times 7$ Gy & Abr./2000 & Ago./2001 & Não \\
50 & IIB & 45 Gy & BATD $4 \times 7$ Gy & Ago./1999 & Jun./2000 & Sim \\
65 & IIIB & 70 Gy & & 1985 & Mar./2000 & Sim \\
71 & - & 60 Gy & BBTD 20 Gy & 1982 & Nov./2000 & Sim \\
41 & IIIB & 50 Gy & BATD $4 \times 7$ Gy & Mar./1999 & Jul./1999 & Não \\
71 & IIA & 42,4 Gy & BBTD & 1988 & Dez./1998 & Não \\
71 & IIA & 45 Gy & BATD $4 \times 7$ Gy & Abr./1999 & Dez./1999 & Não \\
59 & BATD $4 \times 7$ Gy & Dez./1999 & Jan./2000 & Não \\
\hline
\end{tabular}

BATD, braquiterapia de alta taxa de dose; BBTD, braquiterapia de baixa taxa de dose.

Tabela 2 Descrição da técnica de tratamento.

\begin{tabular}{|c|c|l|l|l|l|l|l|l|}
\hline Paciente & $\begin{array}{c}\text { Teleterapia } \\
\text { associada }\end{array}$ & Dose & Isodose & Intervalo & Agulhas & $\begin{array}{c}\text { Dose } \\
\text { bexiga }\end{array}$ & $\begin{array}{l}\text { Relação } \\
150 / 100\end{array}$ & IU \\
\hline 1 & Não & $6 \times 4$ Gy & $85 \%$ & Seis horas & 14 & 215,6 cGy & 13,9 & 2,11 \\
2 & Não & $4 \times 5$ Gy & $85 \%$ & Sete dias & 10 & 198,5 cGy & 24,6 & 1,82 \\
3 & Não & $6 \times 4$ Gy & $85 \%$ & Seis horas & 15 & 233,9 cGy & 23,14 & 1,66 \\
4 & Não & $6 \times 5$ Gy & $85 \%$ & Seis horas & 11 & 294,7 cGy & 24,9 & 1,76 \\
5 & Não & $4 \times 5$ Gy & $85 \%$ & Seis horas & 8 & 132,2 cGy & 18,14 & 1,79 \\
6 & 34 Gy & $4 \times 5$ Gy & $85 \%$ & Seis horas & 10 & 417,7 cGy & 28 & 1,7 \\
7 & Não & $6 \times 5$ Gy & $85 \%$ & Seis horas & 10 & 296,4 cGy & 22,53 & 2,05 \\
8 & 36 Gy & $4 \times 5$ Gy & $85 \%$ & Seis horas & 7 & 239,9 cGy & 19,5 & 1,89 \\
9 & 36 Gy & $4 \times 5$ Gy & $85 \%$ & Seis horas & 20 & 195,1 cGy & 19,25 & 1,65 \\
10 & Não & $4 \times 6$ Gy & $85 \%$ & Seis horas & 5 & 233,5 cGy & 28,6 & 1,59 \\
11 & Não & $4 \times 5$ Gy & $85 \%$ & Sete dias & 6 & 88,2 cGy & 22 & 1,81 \\
\hline
\end{tabular}

IU, índice de uniformidade. 
ginecológicos trimestrais, citologia oncótica e ultra-som pélvico periódicos. Outros exames de imagem (tomografia computadorizada, ressonância magnética) foram realizados para as suspeitas de recidiva, bem como biópsias.

Uma paciente foi a óbito sem avaliação mento.

\section{RESULTADOS}

Dez pacientes $(91 \%)$ tiveram resposta clínica completa, de três a 46 meses.

Três pacientes (27\%) encontravam-se sem evidência de doença, com seguimento de 29 a 44 meses (média de 37 meses). Duas pacientes estão vivas com doença, três morreram pela doença e três perderam o seguimento após a segunda recidiva (sobrevida livre de doença de três a 46 meses; média de 12 meses).

Uma paciente apresentou toxicidade grau III no trato genital (úlcera vaginal) e uma paciente apresentou toxicidade grau II no trato gastrintestinal (retite) (Tabela 3).

\section{DISCUSSÃO}

O tratamento de recorrências de carcinoma do colo do útero é difícil e de pouca eficácia. O índice de sobrevida após recidiva pélvica em cinco anos é de $0 \%$ a $25 \%$, dependendo do local da recidiva e da irradiação anterior ${ }^{\text {(22-26) }}$.

A procura de uma terapia ideal para esses casos é um desafio para cirurgiões, radioterapeutas e oncologistas.

A sobrevida em pacientes que foram submetidas à exenteração pélvica, por de resposta, dois meses após o procedi-

apresentarem doença limitada, é maior do que em pacientes que não puderam ser submetidas à cirurgia. Isto sugere que a melhora do controle local pode aumentar a sobrevida, mesmo porque as pacientes operadas apresentam doença mais limitada e melhores condições clínicas ${ }^{(27-33)}$.

Estudos retrospectivos e de seguimento variável mostram um bom resultado de controle local para neoplasias do colo com recidiva pós-radioterapia, tratadas com braquiterapia intersticial.

O grupo estudado teve um alto índice de resposta completa $(91 \%)$, comparado com os dados da literatura citados. Puthawala et al. obtiveram $67 \%$ e Randall et al., $69 \%$. Quanto à sobrevida livre de doença, o índice foi de $27 \%$, com seguimento de 29 a 44 meses (média de 37 meses), enquanto Randall et al. obtiveram $46 \%$, com seguimento de 24 a 71 meses (média de 59 meses), e Puthawala et al. relataram $33 \%$ em 24 meses de seguimento.

Puthawala et al. descreveram $15 \%$ de toxicidade grau IV (necrose e fístula) e Randall et al. relataram $7,7 \%$ de toxicidade grau IV (fístula reto-vaginal). A série descrita reportou um menor índice de toxicidade: $9 \%$ grau III.

Apesar da complexidade do método, envolvendo vários especialistas, a BATD intersticial para recidivas do colo do útero é um tratamento viável e factível em nosso meio. Apresenta uma relação custo/ benefício vantajosa, pois as pacientes com recidiva causam custos elevados ao sistema de saúde, considerando as interrupções e cuidados paliativos.

Analisando dados da literatura, os resultados obtidos neste estudo, e, principal-

Tabela 3 Resultados do tratamento.

\begin{tabular}{|c|c|c|c|}
\hline Pacientes & Toxicidade & Sobrevida livre de doença & Sobrevida total \\
\hline 1 & - & 3 meses & - \\
2 & - & 31 meses & 54 meses \\
3 & Retite Gll & 3 meses & 14 meses \\
4 & - & 29 meses & 29 meses \\
5 & - & 44 meses & 44 meses \\
6 & - & 5 meses & - \\
7 & Úlcera vaginal & 38 meses & 38 meses \\
8 & - & - & 2 meses \\
9 & - & 5 meses & - \\
10 & - & 46 meses & 35 meses \\
11 & - & 46 meses & 49 meses \\
\hline
\end{tabular}

mente, que essas pacientes não têm proposta terapêutica definida, pode-se afirmar que a BATD intersticial é um tratamento a ser considerado para pacientes selecionadas que apresentarem recidivas pós-radioterapia.

\section{REFERÊNCIAS}

1. Klingerman J. Estimativas sobre incidência e mortalidade por câncer no Brasil em 2001 (Editorial). Rev Bras Cancerol 2001;47(2).

2. Brunschwig A. The surgical treatment of cancer of the cervix: stage I and II. Am J Roentgenol Radium Ther Nucl Med 1968;102:147-51.

3. Park RC, Patow WE, Rogers RE, Zimmerman EA. Treatment for stage I carcinoma of the cervix. Obstet Gynecol 1973;41:117-22.

4. Parker RT, Wilbanks GD, Yowell RK, et al. Radical hysterectomy and pelvic lymphadenectomy with and without preoperative radiotherapy for cervical cancer. A report of 265 patients followed 10 to 22 years. Am J Obstet Gynecol 1967;99:933-43.

5. Pilleron JP, Durand JC, Lenoble JC. Carcinoma of the uterine cervix, stages I and II, treated by radiation therapy and extensive surgery (1000 cases). Cancer 1972;29:593-6.

6. Surwit E, Fowler WC Jr, Palumbo L, Koch G, Gjersten W. Radical hysterectomy with or without preoperative radium for stage IB squamous cell carcinoma of the cervix. Obstet Gynecol 1976;48: 130-3.

7. Walsh JW, Amendola MA, Konerding KF, Tisnado J, Hazra TA. Computed tomographic detection of pelvic and inguinal lymph-node metastases from primary and recurrent pelvic malignant disease. Radiology 1980;137:157-66.

8. Kim RY, Trotti A, Wu C-J, Soong SJ, Salter MM. Radiation alone in the treatment of cancer of the uterine cervix: analysis of pelvic failure and dose response relationship. Int J Radiat Oncol Biol Phys 1989;17:973-8.

9. Fletcher GH. Cancer of the uterine cervix. Janeway lecture, 1970. Am J Roentgenol Radium Ther Nucl Med 1971;111:225-42.

10. Marcial VA, Amato DA, Marks RD, et al. Splitcourse versus continuous pelvis irradiation in carcinoma of the uterine cervix: a prospective randomized clinical trial of the Radiation Therapy Oncology Group. Int J Radiat Oncol Biol Phys 1983;9: 431-6.

11. Perez CA, Breaux S, Madoc-Jones H, et al. Radiation therapy alone in the treatment of carcinoma of the uterine cervix. I. Analysis of tumor recurrence. Cancer 1983;51:1393-402.

12. Kuske RR, Perez CA, Jacobs AJ, et al. Mini-colpostats in the treatment of carcinoma of the uterine cervix. Int J Radiat Oncol Biol Phys 1988;14:899_ 906.

13. Montana GS, Fowler WC, Varia MA, Walton LA, Mack Y, Shemanski L. Carcinoma of the cervix, stage III. Results of radiation therapy. Cancer 1986;57:148-54.

14. Kramer C, Peschel RE, Goldberg N, et al. Radiation treatment of FIGO stage IVA carcinoma of the cervix. Gynecol Oncol 1989;32:323-6.

15. Rubin SC, Hoskins WJ, Lewis JL Jr. Radical hysterectomy for recurrent cervical cancer following radiation therapy. Gynecol Oncol 1987;27:316-24. 16. Roberts WS, Cavanagh D, Bryson, SCP, Lyman GH, 
Hewitt S. Major morbidity after pelvic exenteration: a seven-year experience. Obstet Gynecol 1987;69:617-21.

17. Anthopoulos AP, Manetta A, Larson JE, Podczaski ES, Bartholomew MJ, Mortel L. Pelvic exenteration: a morbidity and mortality analysis of a sevenyear experience. Gynecol Oncol 1989;35:219-23.

18. Murphy WT, Schmitz A. Results of re-irradiation in cancer of the cervix. Radiology 1956;67:37885.

19. Evans Sr Jr, Hilaris BS, Barber HRK. External vs. interstitial irradiation in unresectable recurrent cancer of the cervix. Cancer 1971;28:1284-8.

20. Puthawala AA, Syed AM, Fleming PA, DiSaia PJ. Re-irradiation with interstitial implant for recurren pelvic malignancies. Cancer 1982;50:2810-4.

21. Randall ME, Evans L, Greven KM, McCunniff AJ, Doline RM. Interstitial reirradiation for recurrent gynecologic malignancies: results and analysis of prognostic factors. Gynecol Oncol 1993;48:2331.

22. Haas T, Buchsbaum HJ, Lifshitz S. Nonresectable recurrent pelvic neoplasm. Outcome in patients explored for pelvic exenteration. Gynecol Oncol 1980;9:177-81.

23. Krebs HB, Helmkamp BF, Sevin BU, Poliakoff SR, Nadji M, Averette HE. Recurrent cancer of the cervix following radical hysterectomy and pelvic node dissection. Obstet Gynecol 1982;59:422-7.

24. Chung CK, Nahhas WA, Stryker JA, Mortel R. Treatment outcome of recurrent cervical cancer. J Surg Oncol 1983;24:5-10.

25. Sommers GM, Grigsby PW, Perez CA, et al. Outcome of recurrent cervical carcinoma following definitive irradiation. Gynecol Oncol 1989;35:150 5.

26. Potter ME, Alvarez RD, Gay FL, Shingleton HM, Soong SJ, Hatch KD. Optimal therapy for pelvic recurrence after radical hysterectomy for early-stage cervical cancer. Gynecol Oncol 1990;37:74-7.

27. Ketcham AS, Deckers PJ, Sugarbaker EV, Hoye RC, Thomas LB, Smith RR. Pelvic exenteration for carcinoma of the uterine cervix. A 15-year experience. Cancer 1970;26:513-21.
28. Symmonds RE, Pratt JH, Webb MJ. Exenterative operations: experience with 198 patients. Am J Obstet Gynecol 1975;121:907-18.

29. Morley GW, Lindenauer SM. Pelvic exenterative therapy for gynecologic malignancy: an analysis of 70 cases. Cancer 1976;38(Suppl 1):581-6.

30. Curry SL, Nahhas WA, Jahshan AE, Whitney CW, Mortel R. Pelvic exenteration: a 7-year experience. Gynecol Oncol 1981;11:119-23.

31. Barber HR. Pelvic exenteration. Cancer Invest 1987;5:331-8.

32. Lawhead RA Jr, Clark DG, Smith DH, Pierce VK, Lewis JL Jr. Pelvic exenteration for recurrent or persistent gynecologic malignancies: a 10-year review of the Memorial Sloan-Kettering Cancer Center experience (1972-1981). Gynecol Oncol 1989;33: 279-82.

33. Gemignani ML, Alektiar KM, Leitao M, et al. Radical surgical resection and high-dose intraoperative radiation therapy (HDR-IORT) in patients with recurrent gynecologic cancers. Int J Radiat Oncol Biol Phys 2001;50:687-94. 